\title{
Phenanthrene-Induced Apoptosis and Its Underlying Mechanism
}

\author{
Xiangsheng Hong, ${ }^{\dagger, \ddagger, \perp}$ Jianhui Qin, ${ }^{\dagger}$ Rui Chen, ${ }^{\dagger, \|}$ Lilai Yuan, ${ }^{\dagger, \|}$ Jinmiao Zha, ${ }^{, \dagger, \S \odot ~ C h a o ~ H u a n g, ~}{ }^{\dagger, \S, \perp}$ \\ $\mathrm{Na} \mathrm{Li}{ }^{\dagger, \S}$ Xiaoya Ji, ${ }^{\dagger, \S, \perp}$ and Zijian Wang ${ }^{\dagger, \|}$
}

${ }^{\dagger}$ Key Laboratory of Drinking Water Science and Technology, Research Center for Eco-Environmental Sciences, Chinese Academy of Sciences, Beijing 100085, China

${ }^{\ddagger}$ Key Laboratory of Freshwater Animal Breeding, Ministry of Agriculture, College of Fisheries, Huazhong Agriculture University, Wuhan 430070, China

${ }^{\S}$ Beijing Key Laboratory of Industrial Wastewater Treatment and Reuse, Research Center for Eco-Environmental Sciences, Chinese Academy of Sciences, Beijing 100085, China

"State Key Laboratory of Environmental Aquatic Chemistry, Research Center for Eco-Environmental Sciences, Chinese Academy of Sciences, Beijing 100085, China

${ }^{\perp}$ University of Chinese Academy of Sciences, Beijing 100085, China

Supporting Information

ABSTRACT: Phenanthrene (Phe) is one of the most abundant low-molecular-weight polycyclic aromatic hydrocarbons (PAHs). Widespread human and aquatic organism exposure to Phe has been reported, but the toxic effects of Phe and potential mechanisms are unclear. We focused on the chronic hepatotoxicity of Phe in adult Chinese rare minnows (Gobiocypris rarus) and the underlying mechanisms. The chronic effects of exposing Chinese rare minnows to $8.9,82.3$, or $510.0 \mu \mathrm{g} / \mathrm{L}$ Phe for 30 days were examined by histopathological observation, TUNEL assays, caspase activity assays, and gene expression profiles. The liver lesion frequency and hepatocyte apoptosis were increased in Phe-exposed groups. Caspase 9 and caspase 3 enzyme activity in liver tissues was markedly increased. The expression of miR-17/

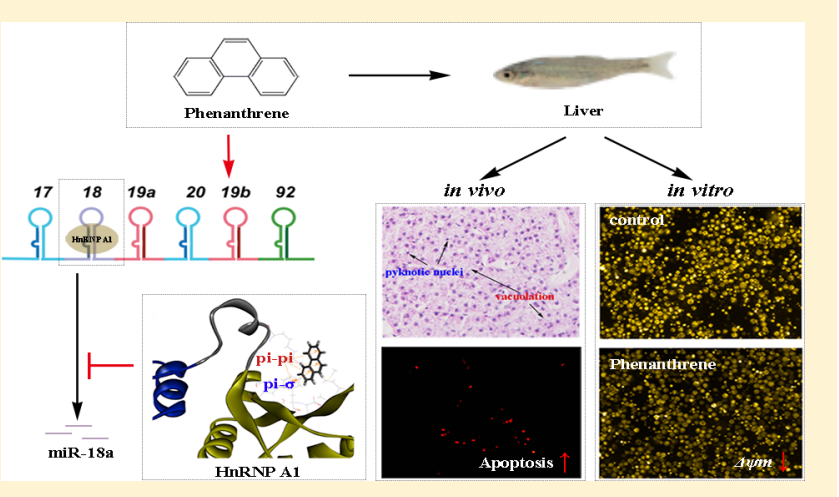
92 cluster members was significantly increased in the 82.3 and $510.0 \mu \mathrm{g} / \mathrm{L}$ groups. Moreover, the response of primary hepatocytes indicated a significant decrease in the mitochondrial membrane potential (MMP) after a 48 h exposure to Phe. Interestingly, miR-18a was significantly decreased in primary hepatocytes in all treatments. Moreover, molecular docking indicated that Phe might have the same binding domain as pri-miR-18a, forming pi-pi and pi- $\sigma$ interactions with heterogeneous nuclear ribonucleoprotein (hnRNP) Al. Given the above, Phe caused liver lesions and induced hepatocyte apoptosis through the intrinsic apoptosis pathway, and the interaction of Phe with hnRNP A1 contributes to the suppression of miR-18a expression and hepatocyte apoptosis.

\section{INTRODUCTION}

Polycyclic aromatic hydrocarbons (PAHs) are a large class of widely distributed environmental pollutants composed of 2-7 fused aromatic rings. ${ }^{1}$ PAHs are divided into two categories according to molecular weight: low-molecular-weight compounds (fewer than 4 rings) and high-molecular-weight compounds ( 4 or more rings). ${ }^{1}$ Because of the involvement of the aryl hydrocarbon receptor (AhR) and cytochrome P4501A (CYP1A) in PAH metabolism, the environmental hazards of high-molecular-weight PAHs are well-known. ${ }^{2-4}$ However, studies of the toxicity of low-molecular-weight PAHs (e.g., phenanthrene) are relatively lacking.

Since 1979, phenanthrene (Phe), one of the most abundant and representative low-molecular-weight PAHs in the environment, ${ }^{5,6}$ has been included in a list of 16 priority PAHs by the United States Environmental Protection Agency (USEPA).
Because Phe is a weak AhR agonist and induces CYP1A activity, the parent compound is most likely toxic. ${ }^{7,8}$ Humans are exposed to Phe through inhalation, dermal absorption, and ingestion. ${ }^{9}$ According to a previous in vitro study, an approximately $24 \%$ human skin absorption rate was detected after $0.64 \mathrm{~cm}^{2}$ of skin was exposed to a $0.1 \mathrm{~mL}$ solution of $1 \mu \mathrm{g} /$ $\mathrm{mL}$ Phe for $24 \mathrm{~h}^{10}$ In The Netherlands, the maximum measured concentration of Phe in drinking water from 120 locations was $6 \mu \mathrm{g} / \mathrm{L} .{ }^{11}$ Phe concentrations in the pore water of the Jiulong River Estuary and Western Xiamen Sea in China are reported to be greater than $20 \mu \mathrm{g} / \mathrm{L}^{12}$

Received: August 7, 2017

Revised: November 3, 2017

Accepted: November 21, 2017

Published: November 21, 2017 
According to previous studies, Phe has been shown to cause embryonic developmental toxicity ${ }^{13}$ and cardiotoxicity, ${ }^{14}$ and it influences spermatogenesis. ${ }^{15}$

The tissue distribution of ${ }^{14} \mathrm{C}$-labeled Phe in Mummichog (Fundulus heteroclitus) indicated that fish liver is a major enrichment organ. ${ }^{16}$ Exposure to $76 \mu \mathrm{g} / \mathrm{L}$ Phe for $96 \mathrm{~h}$ increased the prevalence of histopathological lesions in the liver of African catfish. ${ }^{17}$ Unexpectedly, the Phe concentration in the peripheral venous blood of pregnant women (nonsmokers) and the cord blood of newborns can reach levels as high as $37.61 \pm$ 0.47 and $35.32 \pm 0.34 \mu \mathrm{g} / \mathrm{L}$, respectively. ${ }^{14}$ Moreover, the accumulation of Phe significantly increased reactive oxygen species (ROS) generation and resulted in oxidative stress in the liver of Carassius auratus exposed to $0.05 \mathrm{mg} / \mathrm{L}$ Phe for 4 days. ${ }^{18}$ Although studies have described the effects of Phe in different animals, few studies have focused on the long-term effects of Phe on the fish liver. In addition, the underlying molecular mechanisms linking Phe exposure to liver toxicity are not fully understood.

MicroRNAs (miRNAs) are a group of endogenous, short ( $\sim 22$ nucleotides in length), single-stranded nonprotein-coding RNAs (ncRNAs). ${ }^{19}$ Aberrant expression of miRNAs has been linked to various diseases ${ }^{20}$ and environmental exposures or other causes. ${ }^{21}$ Amelia et al. reported that miR-15 and miR-16 induce apoptosis in leukemic cell lines by down-regulating BCL-2. ${ }^{22}$ Recently, accumulating evidence has indicated a crucial role of miRNA in hepatotoxicant-induced liver injury. ${ }^{23,24}$ miR-17-92 exhibits potent oncogenic activity by repressing c-Myc-induced apoptosis in the E $\mu$-myc Burkitt's lymphoma model. ${ }^{25}$ However, there is no evidence demonstrating the correlation between Phe and miRNA expression in hepatotoxicity.

The main purposes of the current study were to (1) investigate the hepatotoxicity of long-term Phe exposure in the Chinese rare minnow and to (2) attempt to reveal the underlying mechanisms of Phe-induced liver apoptosis.

\section{MATERIALS AND METHODS}

2.1. Chemicals. Phe (purity $97.0 \%$, CAS no. $85-01-8$ ) was purchased from Sigma-Aldrich Corp. (St. Louis, MO). Stock solutions were prepared in acetone and then mixed with distilled water. This solution was diluted with aquarium water, and the final acetone concentration was less than $0.01 \%$.

2.2. Animals. The brood stock of rare minnows was raised in a flow-through system with dechlorinated tap water $(\mathrm{pH}$ 7.2-7.6; hardness 44.0-61.0 $\mathrm{mg} \mathrm{CaCO} / \mathrm{L} ; 25 \pm 1{ }^{\circ} \mathrm{C}$ ) and a photoperiod of $16: 8 \mathrm{~h}$ (light:dark) that has been used to test chemicals in our laboratory for more than 10 years. ${ }^{26}$ Fish were fed a commercial food pellet (Trea, Germany) at a rate of $0.1 \%$ body weight per day and were fed newly hatched brine shrimp (Artemia nauplii) two times daily. The fish were cared for in accordance with the Regulations for the Administration of Affairs Concerning Experimental Animals for the Science and Technology Bureau of China throughout the study.

2.3. Animal Treatments. Six-month-old healthy Chinese rare minnows and offspring from the same pair of brood stock were used in this experiment. The body weights and lengths were $0.69 \pm 0.11 \mathrm{~g}$ and $39.86 \pm 2.5 \mathrm{~mm}$, respectively. Exposure was initiated after 2 weeks of acclimation. Fish were exposed to Phe at concentrations of $10 \mu \mathrm{g} / \mathrm{L}, 100 \mu \mathrm{g} / \mathrm{L}$, and $1000 \mu \mathrm{g} / \mathrm{L}$; the control group received water, and a solvent control group received $0.01 \%$ acetone in water. At each exposure level, 60 fish were randomly separated into three containers. The fish were fed twice daily with newly hatched brine shrimp, and the test solution was refreshed each day during a 30-day exposure period. During the experiment, the water temperature was maintained at $25 \pm 1{ }^{\circ} \mathrm{C}$ with a $\mathrm{pH}$ of $7.0 \pm 0.2$.

2.4. Phe Concentrations in Water. Phe concentrations in the exposure solutions were analyzed using methods reported in a previous study. ${ }^{27}$ Twenty-four hours after the test was initiated, the concentrations (mean \pm standard deviation; \% analyzed/nominal) of Phe in the solutions were $8.9 \pm 0.7$ (89.0\%), $82.3 \pm 5.5(82.3 \%)$, and $510.0 \pm 35.8(51.0 \%) \mu \mathrm{g} / \mathrm{L}$. Actual Phe values are used in subsequent sections.

2.5. Histopathology Analysis. After a 30-day exposure period, the livers of rare minnows (10 fish for each concentration group) were dissected for histopathology. All liver samples were immersed in $4 \%$ paraformaldehyde in 0.1 mol/L phosphate buffer. Fixation was performed at $4{ }^{\circ} \mathrm{C}$ for 48 h. These samples were then transferred to $50 \%$ ethanol and dehydrated in a gradient of ethanol solutions (50-100\%). Finally, liver samples were embedded in paraffin, cut into $5-\mu \mathrm{m}$ thick transverse sections and stained with hematoxylin and eosin (H\&E). Sections were examined, and images were captured under an optical BX53 microscope (Olympus, Tokyo, Japan) (40X). Alterations in the H\&E-stained liver sections were assessed using the method described in our previous study. $^{28}$

2.6. Terminal Deoxynucleotidyl Transferase (TdT) dUTP Nick-End Labeling (TUNEL) Assay and Hoechst 33342 Staining for Apoptosis. From the triplicates of each condition, 10 fish were randomly collected. Paraffin sections were generated as described above. Then, we used the In Situ Cell Death Detection Kit from Roche (Basel, Switzerland) according to the manufacturer's instructions to detect the single- and double-stranded DNA breaks occurring during apoptosis (TUNEL assay). Finally, liver tissues were counterstained with Hoechst 33342 (Sigma Co.) for $5 \mathrm{~min}$ at room temperature. TUNEL-positive nuclei were counted in four nonoverlapping fields per coverslip and then converted to percentages by comparing TUNEL-positive counts with the total cell nuclei determined by Hoechst 33342 counterstaining. ${ }^{29}$ Images were captured using a fluorescence microscope.

2.7. Caspase Activity Assay. After the fish were exposed, triplicate samples (each sample with three fish) in each treatment group were randomly collected. Fish were with MS222 (Sigma), and approximately $50 \mathrm{mg}$ of liver tissues were dissected and homogenized immediately. According to a previous study, ${ }^{30}$ the activities of caspase 3 , caspase 8 , and caspase 9 were determined using the Caspase 3, 8, and 9 activity kit (Beyotime Institute of Biotechnology, China). The results are expressed as the change in enzyme activity relative to the controls.

2.8. Cell Viability. Primary hepatocytes were obtained from normal adult Chinese rare minnows using a modified version of the protocol reported by Ghosh et al. ${ }^{31}$ Details of the primary cell culture and treatment are in the supplement (Supporting Information (SI) Figure S1). Phe cytotoxicity was assessed using the MTT assay. Hepatocytes were seeded in 96-well plates at a density of $5 \times 10^{5}$ cells/well. Phe concentrations ranging from $1-5000 \mu \mathrm{M}$ were prepared for liver cell exposure to determine the optimal concentration. After treatment with DMSO or Phe $(1,10,50,100,500,800$, or $5000 \mu \mathrm{M})$ for $48 \mathrm{~h}$, the culture medium was removed and replaced with a medium containing $0.5 \mathrm{mg}$ MTT dissolved in $1 \times \mathrm{PBS}(\mathrm{pH} 7.2$ ). Four 
hours later, the crystals that had formed were dissolved with $200 \mu \mathrm{L}$ of DMSO. The intensity of the color in each well was measured at a wavelength of $490 \mathrm{~nm}$ using a microplate reader (Figure 4A). Because the fluorescence produced by the high concentration of Phe might interfere with subsequent experiments and the solubility of Phe is limited at higher concentrations, we selected Phe concentrations less than 300 $\mu \mathrm{M}$ for treatments in subsequent experiments.

2.9. Mitochondrial Membrane Potential $\left(\Delta \psi_{\mathrm{m}}\right)$. Hepatocytes were exposed to either DMSO or Phe $(1,10,50,100$, $300 \mu \mathrm{M})$ for $48 \mathrm{~h}$ after 3 days of culture. because tetramethylrhodamine ethyl ester (TMRE) is extensively used to label and measure MMP in cells, ${ }^{32}$ hepatocytes were washed twice with ice-cold $1 \times$ PBS and immersed in TMRE (working concentration: $50 \mathrm{nM}$ ) for $30 \mathrm{~min}$ and Hoechst 33342 (working concentration: $10 \mu \mathrm{g} / \mathrm{mL}$ ) for $10 \mathrm{~min}$ at $25{ }^{\circ} \mathrm{C}$ (Sigma). The intensity of the colors in each well was measured at wavelengths of 573 and $350 \mathrm{~nm}$ using a High Content Screening Microplate Imaging Reader (PerkinElmer).

2.10. QPCR Analysis. Total RNA from dissected organs after 30 days of Phe exposure or from primary hepatocytes treated with Phe for $48 \mathrm{~h}$ ( $n=$ three replicates per treatment) was isolated using SV Total RNA Isolation kits and reverse transcribed into cDNA using M-MLV Reverse Transcriptase (Promega). mRNA expression was measured with an ABI 7500 real-time quantitative PCR system (Life Technologies, USA) using a previously described method. ${ }^{33}$ The miRNAs were isolated using a miRcute miRNA Isolation Kit (TIANGEN, Beijing, China) and reverse-transcribed into cDNAs using the miRcute miRNA First-Strand cDNA Synthesis Kit (TIANGEN, Beijing, China). miRNA expression was measured by qPCR using the miRcute miRNA qPCR Detection Kit (TIANGEN, Beijing, China) with an ABI 7500 real-time quantitative PCR system. $^{34}$ All processes described above were performed according to the manufacturers' instructions. The primer pairs are listed in SI Table S1, and $\beta$-actin and 5 S rRNA ${ }^{35}$ were used as endogenous controls for mRNA and miRNA, respectively. The PCR efficiencies ranged from $93.6 \%$ to $102.5 \%$, and the results were analyzed using the $2^{-\Delta \Delta \mathrm{Ct}}$ method. ${ }^{36}$

2.11. Molecular Docking. The crystal structure of the heterogeneous nuclear ribonucleoprotein (hnRNP) A1 protein bound to $\mathrm{RNA}^{37}$ was extracted from the RCSB Protein Data Bank (PDB, http://www.rcsb.org/pdb). SI Section 1 contains a description of homologous modeling and model assessment procedures. The molecular docking analysis was implemented according to our previous study. ${ }^{38}$ All docking experiments were performed using the Discovery Studio 4.0 software package (Accelrys Software Inc., San Diego, CA). The protocol was generated using the CDOCKER protocol.

2.12. Statistical Analysis. Statistically significant differences between the control and exposure groups were assessed using one-way analysis of variance (ANOVA) followed by Tukey's test with SPSS software (version 22.0). All results are expressed as the mean \pm standard error of the mean (S.E.M.). Significant differences between the treatment groups and the corresponding control group are indicated by $*$, indicating $p<$ 0.05 .

\section{RESULTS}

Effect of Phe on Liver Tissues In Vivo. 3.1. Death and Growth. During exposure, none of the fish in any of the treatment groups died. However, during the first 3 days of exposure, 90\% (54/60) of fish that received the $510.0 \mu \mathrm{g} / \mathrm{L}$ treatment suffered different degrees of narcotic effects, such as body rolling and sinking to the bottom; nonetheless, their gills remained in motion. On the fourth day of exposure, all fish resumed normal swimming behavior. In addition, the body lengths and body weights of fish in the treatment groups were not significantly different from the control group (data not shown).

3.2. Liver Histopathological Lesions. The livers from the controls had a normal appearance, including a normal structure, an occasional vacuole, and a similar prevalence of hepatocytes (Figure 1A). Compared with the control group, the Chinese

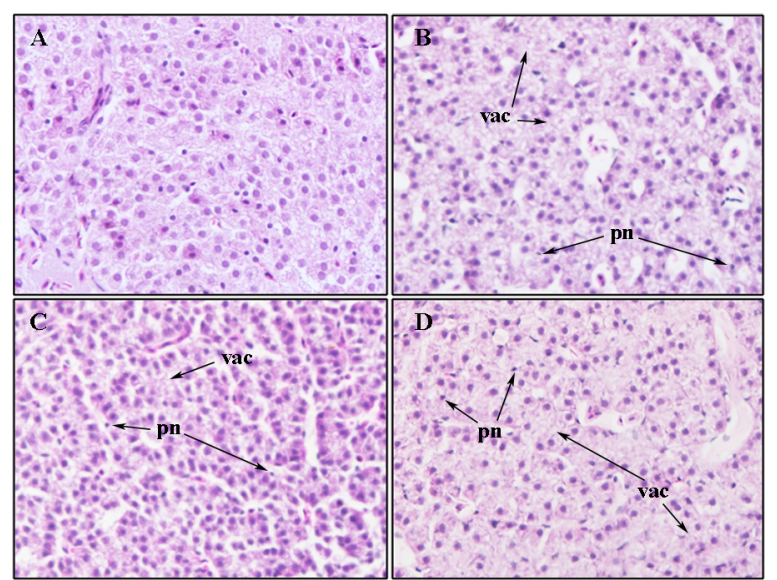

Figure 1. Photomicrographs of H\&E-stained paraffin sections showing the liver pathology in adult rare minnows 30 days after exposure to 0 $\mu \mathrm{g} / \mathrm{L}(\mathrm{A}), 8.9 \mu \mathrm{g} / \mathrm{L}(\mathrm{B}), 82.3 \mu \mathrm{g} / \mathrm{L}(\mathrm{C})$, or $510.0 \mu \mathrm{g} / \mathrm{L}$ (D) Phe. Compared to the control treatment, fish exposed to different Phe concentrations displayed similar histopathological changes, including pyknotic nuclei (pn) and vacuolation (vac). Magnification 40×; Stained with $\mathrm{H} \& \mathrm{E}$.

rare minnows exposed to Phe $(8.9,82.3$, or $510.0 \mu \mathrm{g} / \mathrm{L})$ exhibited a significant induction in liver histopathological lesions, including pyknotic nuclei and vacuolation, after 30 days of exposure (Figure 1B, C and D).

3.3. Phe Induced Apoptosis in Liver Tissues of the Rare Minnow. As shown in Figure 2, apoptosis was significantly increased in liver tissues after adult Chinese rare minnows were exposed to $82.3 \mu \mathrm{g} / \mathrm{L}$ or $510.0 \mu \mathrm{g} / \mathrm{L}$ Phe for 30 days (Figure $2 \mathrm{~B}, p<0.05$ ). However, no significant differences were observed between the group receiving the $8.9 \mu \mathrm{g} / \mathrm{L}$ treatments and the control (Figure 2B).

3.4. Phe Exposure Induced Caspase 3 and 9 Activity. Caspase 3 activity was markedly elevated in the liver after treatment (Figure 3A, $p<0.05$ ). In contrast, no significant upregulation of caspase 8 activity was observed after Phe treatment (Figure 3A). In addition, caspase 9 activity was only significantly increased by the $510.0 \mu \mathrm{g} / \mathrm{L}$ treatment (Figure 3A, $p<0.05$ ).

3.5. Expression of Genes Related to the p53 Pathway. A significant up-regulation of p53 mRNA was observed in the groups treated with $8.9 \mu \mathrm{g} / \mathrm{L}$ or $82.3 \mu \mathrm{g} / \mathrm{L}$ Phe (Figure $3 \mathrm{~B}, p<$ $0.05)$. A strong induction in $p 21$ and gadd45 $\alpha$ mRNA was observed in all treatment groups (Figure $3 \mathrm{~B}, p<0.05$ ). The transcript levels of the pro-apoptotic protein bax (the bcl-2associated $\mathrm{x}$ protein) were significantly up-regulated by 82.3 $\mu \mathrm{g} / \mathrm{L}$ and $510.0 \mu \mathrm{g} / \mathrm{L}$ Phe, with 1.82-fold and 3.96-fold increases, respectively (Figure 3B, $p<0.05$ ). The mRNA levels of the antiapoptotic protein $b c l-2$ were only significantly up- 

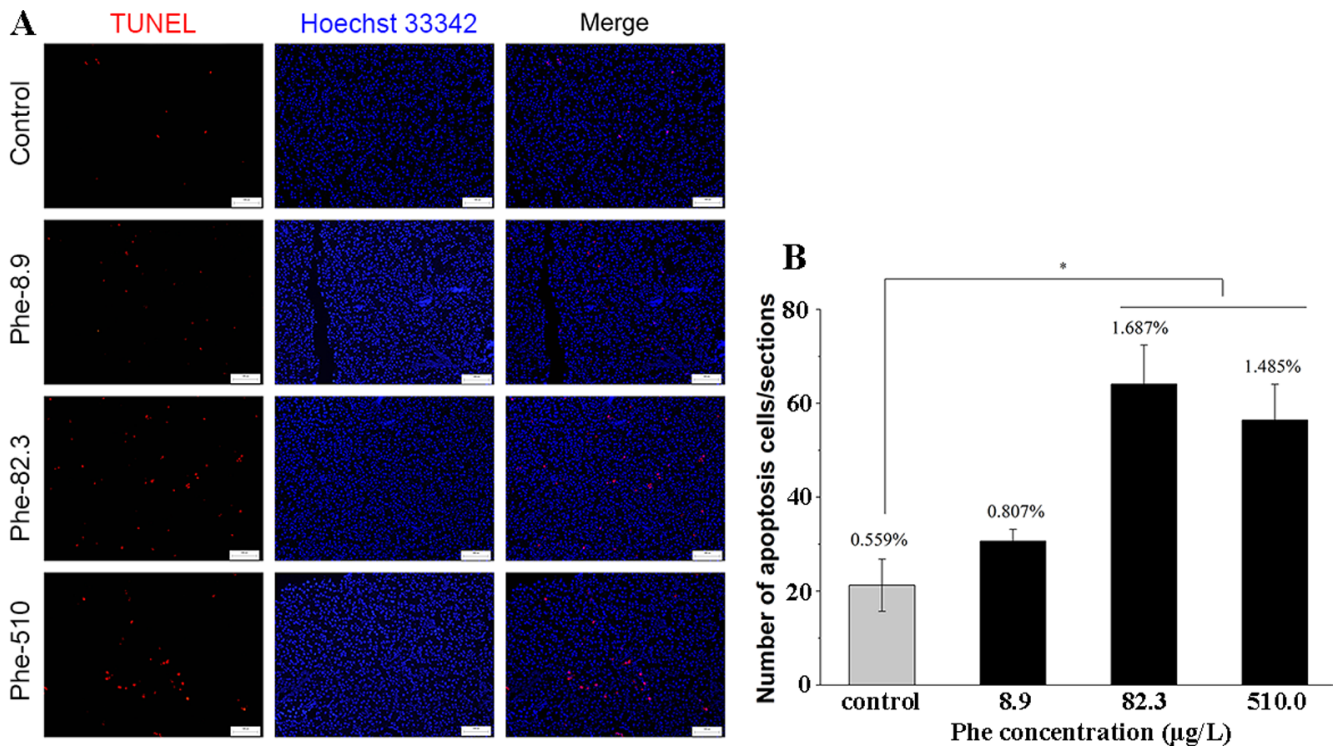

Figure 2. Phe induces apoptosis in the liver tissues of Chinese rare minnows. (A) TUNEL and Hoechst staining were performed as described in the Materials and Methods section. Representative images of TUNEL-positive cells (red, first column) and Hoechst 33342 counterstaining (blue, middle column). The induction of apoptosis was assessed using fluorescence microscopy, bars $=100 \mu \mathrm{m}$. (B) Number of TUNEL-positive hepatocytes relative to Hoechst-stained hepatocytes. Data are presented as the mean \pm SEM for each treatment $(n=10$ fish for each concentration group). Error bars represent $95 \%$ CI. $* p<0.05$ compared to the control.
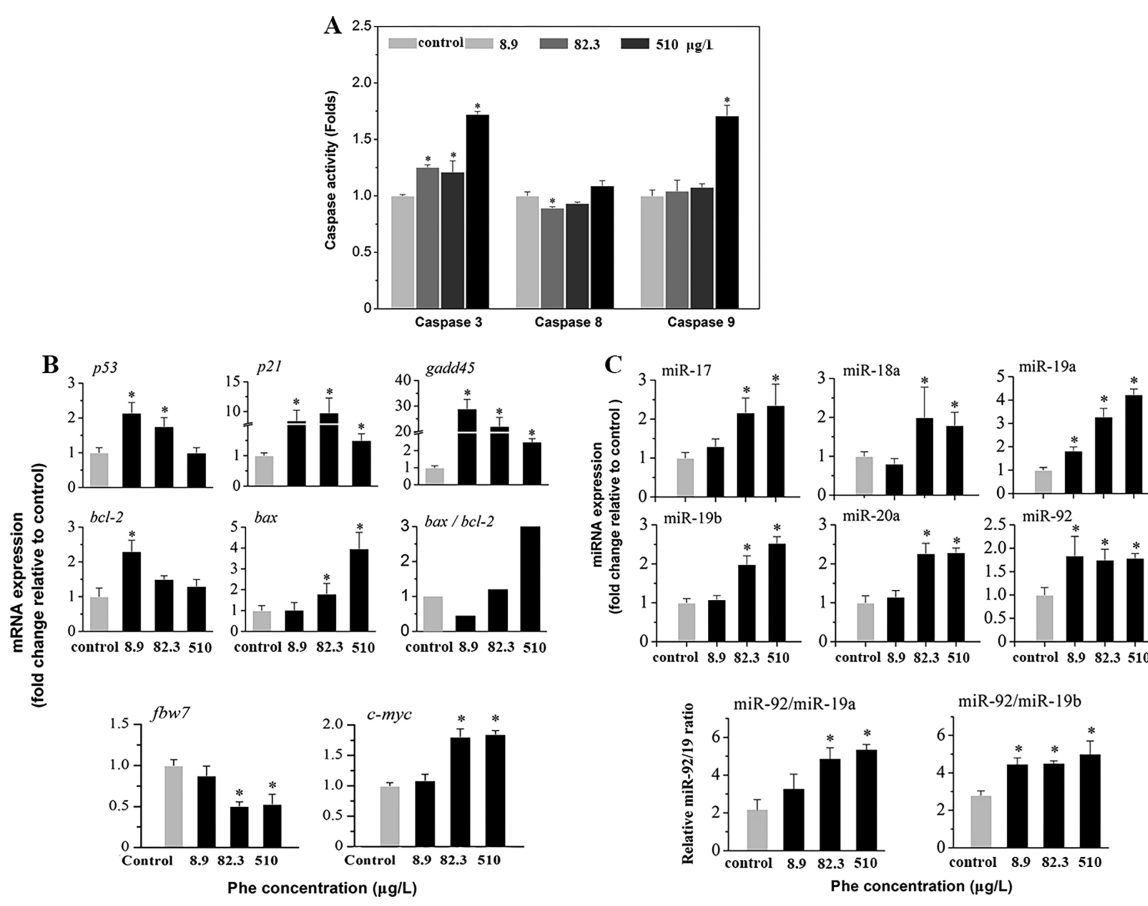

Figure 3. Phe induces apoptosis through the mitochondrial pathway. (A) Caspase 3, caspase 8, and caspase 9 activity in the liver of Chinese rare minnows that were exposed to Phe for 30 days. Values were normalized to the control. (B) Relative expression levels of $p 53, p 21, g a d d 45 \alpha, b c l-2$, and bax mRNAs in Chinese rare minnows after 30 days of exposure to Phe. Bars represent the relative mRNA expression levels in exposed fish versus control fish. $\beta$-actin was used for normalization. (C) Effects of 30 days of Phe exposure on the expression levels of the miR-17/92 cluster. Bars represent the relative miRNA expression levels in exposed fish versus control fish. The 5S rRNA was used for normalization. All values are presented as the mean $\pm \operatorname{SEM}(n=3)$. The asterisk indicates a significant difference from controls $(p<0.05)$, as assessed by ANOVA followed by Tukey's test.

regulated by the $8.9 \mu \mathrm{g} / \mathrm{L}$ treatment (Figure $3 \mathrm{~B}, p<0.05$ ). As shown in Figure $^{3} \mathrm{~B}, 82.3 \mu \mathrm{g} / \mathrm{L}$ and $510 \mu \mathrm{g} / \mathrm{L}$ treatments significantly decreased the expression of $f b w 7$ and increased the $c$-myc expression levels $(p<0.05)$.

3.6. Phe Induced Changes in miRNA Expression in the Liver. As shown in Figure 3C, the expression levels of miR-17, miR-18a, miR-19a, miR-19b, and miR-20a were significantly up- regulated by the $82.3 \mu \mathrm{g} / \mathrm{L}$ and $510.0 \mu \mathrm{g} / \mathrm{L}$ treatments (Figure $3 \mathrm{C}, p<0.05)$. In the low-concentration group, only miR-19a and miR-92 expression levels were notably induced (Figure 3C, $p<0.05)$. Moreover, miR-19a and miR-92 expression levels were notably induced by all treatments (Figure 3C, $p<0.05$ ).

Effects of Phe on Primary Hepatocytes. 3.7. Mitochondrial Membrane Potential. Hepatocytes were treated with 
$0.1 \%$ DMSO or $1-300 \mu \mathrm{M}$ Phe and collected to detect the MMP. A decrease in the MMP is an iconic event in the early stages of apoptosis. As shown in Figure 4B, the MMP was only
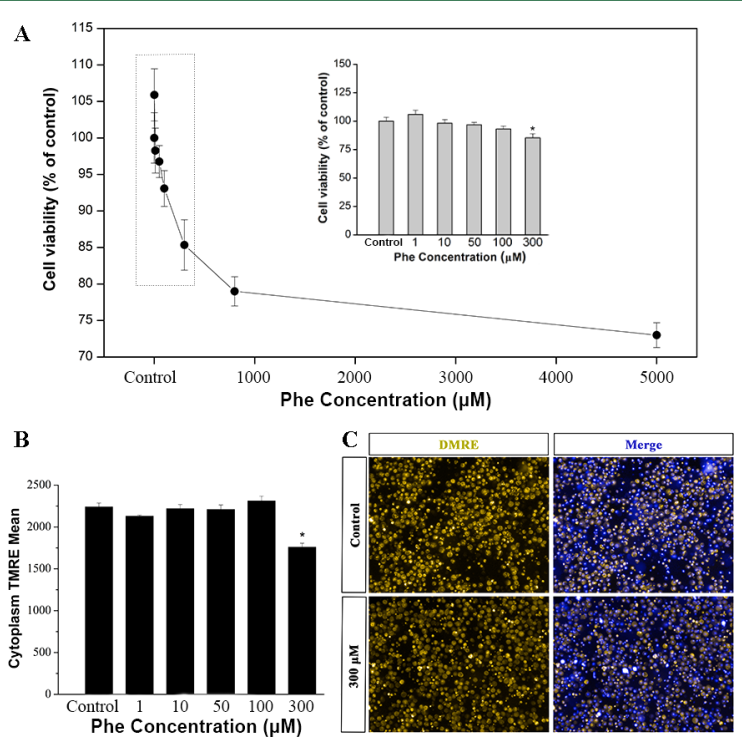

Figure 4. Effects of Phe on cell viability and mitochondrial membrane potential $\left(\Delta \psi_{\mathrm{m}}\right)$ in Chinese rare minnow hepatocytes. (A) The viability of cells treated with various concentrations of Phe $(1-5,000$ $\mu \mathrm{M}$ ) for $48 \mathrm{~h}$ was determined using MTT assays. (B) The mitochondrial membrane potential of hepatocytes treated with increasing concentrations $(1-300 \mu \mathrm{M})$ of Phe for $48 \mathrm{~h}$. (C) The illustration shows the control and the group treated with the highest Phe concentration after staining with TMRE (data not shown for other groups). Scale bars represent $50 \mu \mathrm{m}$. The results are presented as the mean \pm SEM of triplicates.

markedly decreased in the group treated with $300 \mu \mathrm{M}$ Phe $(p<$ 0.05). Compared with the control, the $1-100 \mu \mathrm{M}$ Phe treatments did not induce obvious hepatocyte apoptosis, whereas significant hepatocyte apoptosis was observed at 300 $\mu \mathrm{M}$ Phe (data not shown).

3.8. Phe Induced Changes in miRNA and mRNA Expression in Liver Cells. The miR-17/92 cluster participates in the regulation of apoptotic signaling. In this study, the expression of this cluster in vitro was very different from its expression in vivo, particularly for miR-18a, which was strongly repressed in all exposure groups (Figure 5A, $p<0.05$ ). DGCR8, Drosha, Dicer, and hnRNP Al are involved in miRNA biosynthesis. ${ }^{39,40}$ Therefore, we detected the expression of DGCR8, Drosha, Dicer, and hnRNP A1 by qPCR to identify the mechanism underlying the differences in the expression of the miR-17/92 cluster. However, none of these mRNAs exhibited a significant change (Figure 5B).

3.9. Docking Analysis. Based on the results of the model assessment, no irrational conformation was observed for the constructed UP1 domain model (SI Figure S2). Figure 6 shows the docking view of Phe in the RNA binding site of hnRAP A1. Phe docked into hnRNP Al with its hydrophobic ring located in the inner region of the binding pocket (RRM1) of the human, zebrafish, and Chinese rare minnow protein (Figure 6). The main mode of interaction involves the aromatic ring of Phe interacting with lysine (LYS) 8 and arginine (ARG) 85 at the binding site and forming pi-pi and pi- $\sigma$ interactions (Figure 6C).

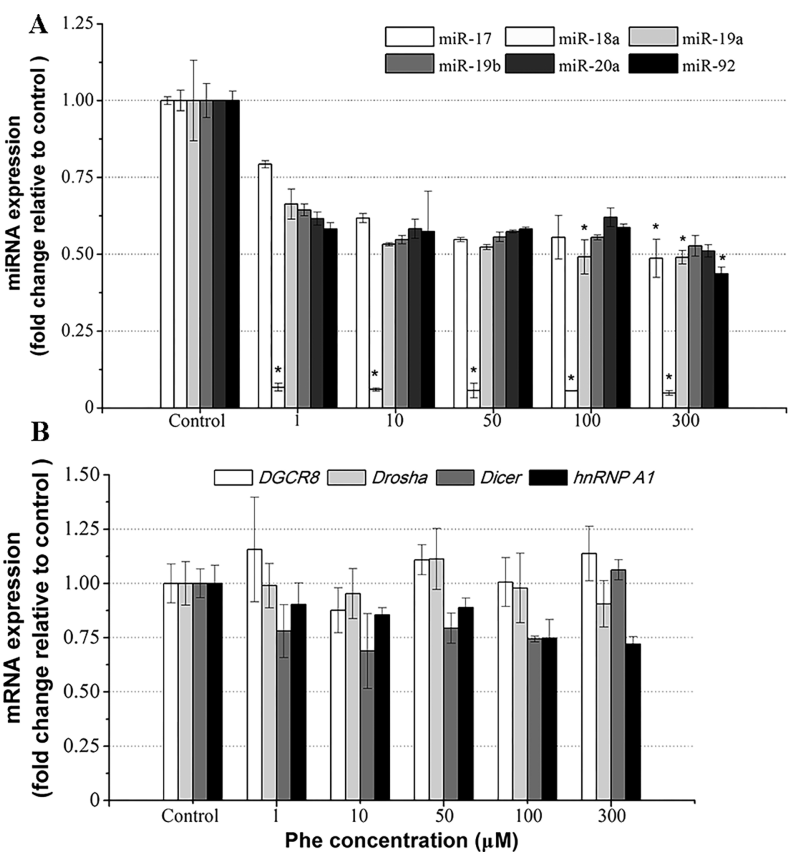

Figure 5. Changes in miRNA and mRNA expression in Chinese rare minnow hepatocytes exposed to Phe for $48 \mathrm{~h}$. (A) Expression of the miR-17/92 cluster after Phe treatment. (B) Expression of genes associated with the miRNA biosynthesis pathway. Error bars represent $95 \%$ CI. $* p<0.05$ compared to the control.

\section{DISCUSSION}

Phe, one of the most abundant low-weight PAHs in the atmosphere $^{41}$ and aquatic environments, is produced from petrogenic and pyrogenic sources. ${ }^{42}$ However, due to the simple structure and lipophilicity of low-molecular-weight PAHs, generally consisting of only carbon and hydrogen, these compounds are usually thought to have low baseline toxicity compared with high-molecular-weight PAHs. Similar to the Phe toxicity observed in this study, our recent study showed that only $1 \mu \mathrm{g} / \mathrm{L} \mathrm{BaP}$ exposure induced significant liver apoptosis in Chinese rare minnows. ${ }^{30}$ Previous literature describing the toxicity and carcinogenicity of low-molecularweight PAHs is relatively limited. Therefore, in this study, Phe was selected as a representative low-molecular-weight PAH.

During the early stages of exposure, fish in the groups treated with the highest Phe concentration displayed symptoms of narcosis, consistent with the results of previous studies. ${ }^{43}$ All fish exhibited normal behaviors approximately 3-4 days later. Although the liver serves as a major site of PAH metabolism in fish, current studies on Phe have demonstrated that Phe is a weak AhR agonist and CYP1A activity inducer. ${ }^{27,44}$ Therefore, the narcosis symptoms were more likely due to the parent compound accumulation of Phe in hepatic tissues. ${ }^{44}$

In Phe-exposed fish, the induction of lesions (Figure 1) and apoptosis (Figure 2) was observed in liver tissues after 30 days of exposure and was accompanied by increased local Phe concentrations. Similarly, pyknotic nuclei, a typical morphological feature of apoptosis, were observed in the liver tissues of juvenile African catfish (Clarias gariepinus) exposed to Phe for $96 \mathrm{~h}(6.2 \mu \mathrm{g} / \mathrm{L}$ and $76 \mu \mathrm{g} / \mathrm{L}) .{ }^{17}$ In another in vitro study combining human oligonucleotide chips and bioinformatics analyses, Song et al. $^{45}$ identified apoptosis as one of the key biological processes related to hepatotoxicity in hepatocytes (HepG2) after a 48-h exposure to $500 \mu \mathrm{M}$ Phe. Furthermore, 


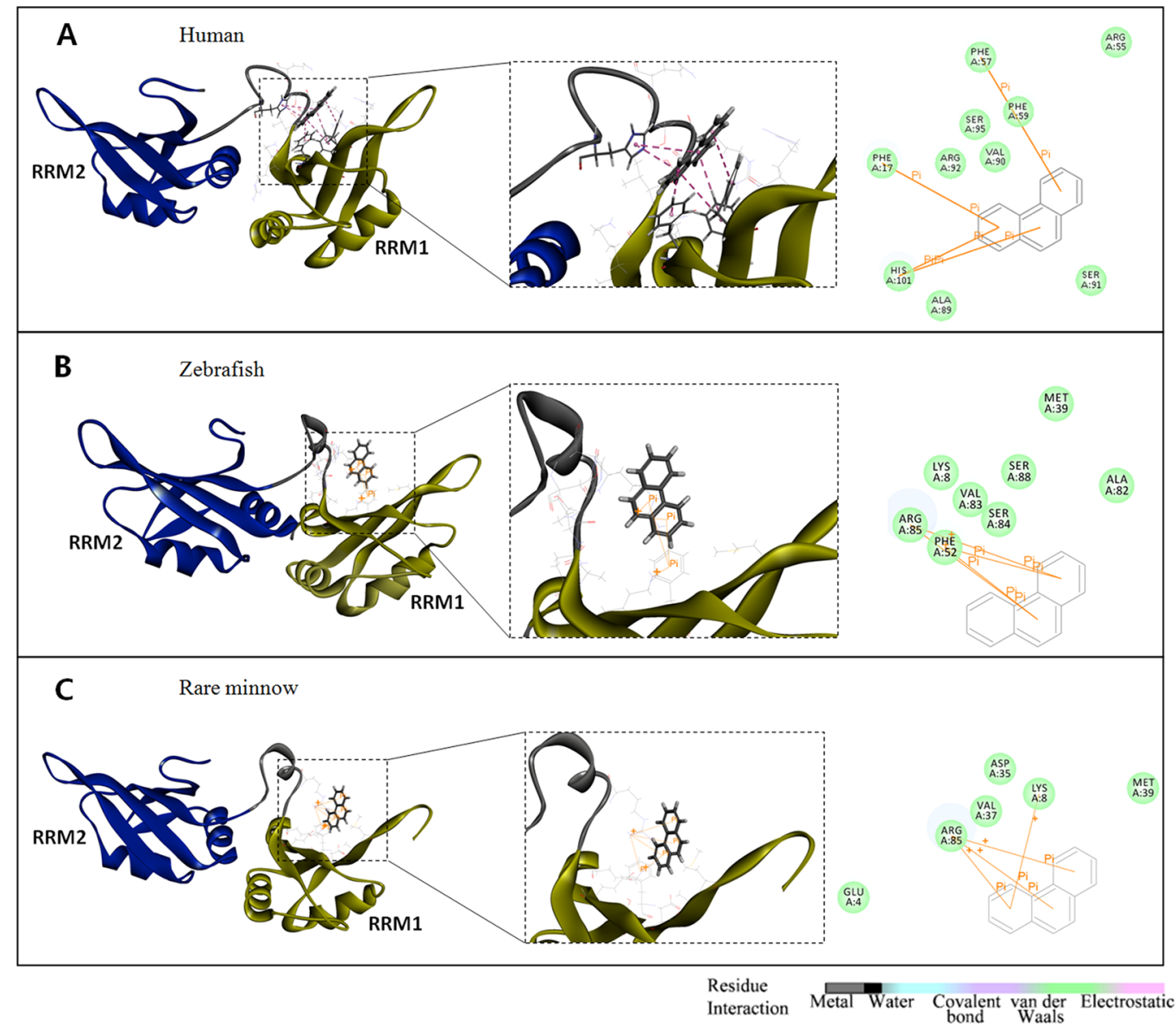

Figure 6. Molecular docking analysis of Phe with the RNA recognition motif domain of hnRNP A1. Ribbon diagram of UP1 (RRM1 marked in yellow; linker marked in gray; RRM2 marked in blue) bound to Phe in the RRM1 domain of hnRNP A1. (A) Best docking mode of Phe (shown as sticks); the rings of Phe interact with phenylalanine (PHE) 17, PHE 57, and histidine (HIS) 101 of the human hnRNP A1 protein. (B) Best docking mode of Phe (shown as sticks); the rings of Phe interact with PHE 52 and ARG 85 of the zebrafish hnRNP A1 protein. (C) Best docking mode of Phe (shown as sticks); the rings of Phe interact with LYS 8 and ARG 85 of the Chinese rare minnow hnRNP A1 protein.

toxic effects of low-molecular-weight PAHs (e.g., Phe) have been observed, even at environmentally relevant concentrations. ${ }^{15,46}$

Apoptosis is crucial for the maintenance of liver health. The mechanisms of apoptosis are highly complex and sophisticated, and two main apoptotic pathways are known-the extrinsic pathway and the intrinsic pathway. ${ }^{47,48}$ Based on the results of a previous transcriptome sequencing analysis, high-molecularweight PAHs (e.g., $3 \mu \mathrm{M} \mathrm{BaP}$ ) induced the expression of genes associated with apoptosis in HepG2 cells. ${ }^{49}$ Moreover, $5 \mu \mathrm{M}$ $\mathrm{BaP}$ induced the apoptotic death of mouse hepatoma Hepalclc7 cells through the activation of the intrinsic pathway. ${ }^{50}$ However, the mechanism by which low-molecularweight Phe induces liver cell apoptosis is not well explained. Here, Phe-induced apoptosis in liver tissues was preceded by increased bax mRNA and the activation of caspase 9 and caspase 3 (Figure $3 \mathrm{~A}$ and $3 \mathrm{~B}$ ). Thus, we speculate that Pheinduced hepatocyte apoptosis may be directly related to the mitochondrial pathway. Consistent with this hypothesis, the high-molecular-weight $\mathrm{PAH} \mathrm{BaP}$ induced apoptosis in hepatoma cells by activating both the extrinsic pathway and the intrinsic pathway, as determined through a microarray analysis. ${ }^{49}$ Based on the combined mechanisms by which highmolecular-weight $\mathrm{PAHs}^{50}$ and low-molecular-weight PAHs (Phe) induce apoptosis, both types of $\mathrm{PAH}$ induce apoptosis through the mitochondrial pathway. Moreover, in the present study, the highest concentration of Phe did not affect the levels of p53 mRNA. These findings were consistent with previous studies showing that $\mathrm{BaP}$ induced $\mathrm{p} 53$ phosphorylation and a block at the G2 phase of the cell cycle in JEG-3 cells. ${ }^{51}$ Furthermore, low concentrations of Phe significantly increased the expression of $p 21$ and gadd45 $\alpha$ mRNA (a regulator of the cell cycle and a gene involved in cell repair, respectively). ${ }^{52,53}$ According to the hematoxylin and eosin (H\&E) staining presented in a recent study, a low concentration of Phe $(6.2$ $\mu \mathrm{g} / \mathrm{L}$ ) only induced a few pyknotic nuclei (a morphological marker of apoptosis). ${ }^{17}$ In our study, a low concentration of Phe $(8.9 \mu \mathrm{g} / \mathrm{L})$ did not cause significant apoptosis. As the Phe concentrations increased, the number of apoptotic cells significantly increased, consistent with a previous study. ${ }^{17}$

Large numbers of miRNAs involved in regulating the apoptosis pathways have been reported. ${ }^{54}$ The response profiles of apoptotic pathway-related miRNAs were observed in our study. Interestingly, in this study, overexpression of the miR$17 / 92$ cluster was a relatively more sensitive indicator of liver apoptosis responses in vivo. As shown in a study by Lima et al., alterations in the expression of the miR-17/92 cluster are associated with responses in apoptosis pathways. ${ }^{55} \mathrm{Fbw} 7$ (F-box and WD repeat domain-containing 7) was previously validated as the target of miR-92, which depresses c-myc-induced 
apoptosis, ${ }^{56}$ and $c-m y c$ ( $c-m y c$ proto-oncogene) was shown to be the target of miR-19, which depresses c-myc-induced proliferation. ${ }^{57}$ Olive et al. ${ }^{58}$ found that $c-m y c$ expression was induced by miR-92 overexpression, partially by repressing $f b w 7$, whereas miR-19 repressed $c$-myc-induced apoptosis. Our results for $f b w 7$ and $c-m y c$ expression in the $82.3 \mu \mathrm{g} / \mathrm{L}$ and $510.0 \mu \mathrm{g} / \mathrm{L}$ groups agreed with previous studies. Although miR-19 expression represents an antagonistic relationship with the observed decrease of $f b w 7$ and the $c-m y c$-induced apoptosis, an increase in the miR-92:miR-19 ratio is likely to favor $c-m y c$ induced apoptosis. ${ }^{58}$ In our study, an obvious increase in the miR-92:miR-19 ratio was observed, which was in line with the TUNEL assay results in response to $82.3 \mu \mathrm{g} / \mathrm{L}$ and $510.0 \mu \mathrm{g} / \mathrm{L}$ Phe.

Because hepatocytes have been widely used to study the hepatotoxicity of chemicals in vitro, ${ }^{59}$ primary hepatocytes of Chinese rare minnows were used to study the hepatotoxicity and toxicity mechanisms in vitro. First, the cytotoxicity of Phe was assessed using the MTT assay to determine an optimal concentration. Hepatocyte apoptosis was significantly induced at $300 \mu \mathrm{M}$ Phe after $48 \mathrm{~h}$ (data not shown). Mitochondrial dysfunction is associated with the induction of apoptosis and is a key indicator of cell health or lesions. ${ }^{60,61}$ Thus, we validated the changes in the MMP in hepatocytes using TMRE/Hoechst 33342 staining. ${ }^{32}$ In the present study, a significant mitochondrial membrane depolarization (decreased $\Delta \psi_{\mathrm{m}}$ ) of hepatocytes was also observed at $300 \mu \mathrm{M}$ Phe beginning after $48 \mathrm{~h}$ of exposure. The primary hepatocyte assay indicated that Phe induced mitochondrial-mediated apoptosis, which agreed with in vivo data. In primary hepatocyte assays, the downregulation of miR-17, miR-18a, miR-19a, and miR-92 of hepatocytes was observed with $300 \mu \mathrm{M}$ Phe treatment (Figure $5 A)$, which is consistent with a previous study. ${ }^{21}$ Three key genes (DGCR8, Drosha, and Dicer) that participate in the miRNA biogenesis pathway were detected using qPCR to explore the potential reasons for miRNA down-regulation in in vitro experiments (Figure 5B). ${ }^{62}$ Interestingly, the miR-18a expression levels were very significantly down-regulated in all exposure groups (Figure 5A). As shown in the study by Fujiya et al., ${ }^{63} \mathrm{miR}-18 \mathrm{a}$ overexpression induced obvious apoptosis in SW620 cells. Accordingly, an interesting possibility for the lack of a significant alteration in hepatocyte apoptosis would be the extremely significant down-regulation of miR-18a.

HnRNP A1, an RNA-binding protein (RBP), is required for pri-miR-18a processing. ${ }^{40}$ The RNA recognition motifs (RRMs) of hnRNP A1 and its binding site in RNA 5'-AGU$3^{\prime}$ have been determined by resolving the crystal structure (4YOE). ${ }^{37}$ The RRMs are located between amino acids 1 and 200 of the protein sequence, and the similarity of the RRMs between species is greater than $90 \%$ (data not shown). Therefore, in this study, the 4YOE RNA binding site was used to perform homology modeling, assess the model, and perform molecular docking simulations with Phe. The hnRNP A1 protein binds to the loop of pri-miR-18a and induces relaxation at the stem. ${ }^{64}$ This relaxed structure is a more favorable cleavage site for Drosha. ${ }^{65}$ According to a comparison of the amino acid sequence homology, Chinese rare minnow, zebrafish, and human hnRNP A1 share more than 90\% similarity in the RNA-binding domain (RBD). ${ }^{65}$ Although $h n R N P A 1$ expression was decreased by each dose of Phe without achieving significance, the hydrophobic ring of Phe was shown to form pi-pi and pi- $\sigma$ interactions with the RRM1 domain of hnRNP A1 based on homologous modeling and molecular docking methods (Figure 6). Therefore, this antagonism may inhibit the hnRNP Al-induced post-transcriptional cleavage of pri-miR-18a and thereby decrease miR-18a expression.

\section{ASSOCIATED CONTENT}

\section{S Supporting Information}

The Supporting Information is available free of charge on the ACS Publications website at DOI: 10.1021/acs.est.7b04045.

(1) Table S1. Primers for quantitative real-time PCR analysis; (2) Figure S1. Primary liver cell culture and treatment; and (3) Figure S2. Homologous modeling and model assessment of hnRNP Al (PDF)

\section{AUTHOR INFORMATION}

\section{Corresponding Author}

*Phone: 86-10-62849107; e-mail: jmzha@rcees.ac.cn or jinmiaozha@gmail.com.

ORCID

Jinmiao Zha: 0000-0003-4928-8992

Notes

The authors declare no competing financial interest.

\section{ACKNOWLEDGMENTS}

We are grateful to the National Natural Science Foundation of China (21677165) for financial support. The financial support from the Major International Joint Research Project of the National Natural Science Foundation of China (51420105012) is also highly appreciated.

\section{REFERENCES}

(1) Juhasz, A. L.; Naidu, R. Bioremediation of high molecular weight polycyclic aromatic hydrocarbons: a review of the microbial degradation of benzo[a]pyrene. Int. Biodeterior. Biodegrad. 2000, 45 (1-2), 57-88.

(2) Garner, L. V. T.; Giulio, R. Di. AHR2 knockdown prevents PAHmediated cardiac toxicity and XRE- and ARE-associated gene induction in zebrafish (Danio rerio. Toxicol. Appl. Pharmacol. 2011, 254 (3), 280-287.

(3) James, K.; Peters, R. E.; Laird, B. D.; Ma, W. K.; Wickstrom, M.; Stephenson, G. L.; Siciliano, S. D. Human Exposure Assessment: A Case Study of 8 PAH Contaminated Soils Using in Vitro Digestors and the Juvenile Swine Model. Environ. Sci. Technol. 2011, 45 (10), $4586-4593$.

(4) Santacroce, M. P.; Pastore, A. S.; Tinelli, A.; Colamonaco, M.; Crescenzo, G. Implications for chronic toxicity of benzo[a]pyrene in sea bream cultured hepatocytes: Cytotoxicity, inflammation, and cancerogenesis. Environ. Toxicol. 2015, 30 (9), 1045-1062.

(5) Dong, T. T. T.; Lee, B.-K. Characteristics, toxicity, and source apportionment of polycylic aromatic hydrocarbons (PAHs) in road dust of Ulsan, Korea. Chemosphere 2009, 74 (9), 1245-1253.

(6) Gregoris, E.; Argiriadis, E.; Vecchiato, M.; Zambon, S.; De Pieri, S.; Donateo, A.; Contini, D.; Piazza, R.; Barbante, C.; Gambaro, A. Gas-particle distributions, sources and health effects of polycyclic aromatic hydrocarbons (PAHs), polychlorinated biphenyls (PCBs) and polychlorinated naphthalenes (PCNs) in Venice aerosols. Sci. Total Environ. 2014, 476-477, 393-405.

(7) Hawkins, S. a.; Billiard, S. M.; Tabash, S. P.; Stephen Brown, R.; Hodson, P. V. Altering Cytochrome P4501a Activity Affects Polycyclic Aromatic Hydrocarbon Metabolism and Toxicity in Rainbow Trout (Oncorhynchus Mykiss). Environ. Toxicol. Chem. 2002, 21 (9), 18451853.

(8) Billiard, S. M.; Hodson, P. V.; Bols, N. C. Does the potency of polycyclic aromatic hydro-carbons (PAHs) for inducing CYP1A1 in 
juvenile trout (Oncorhynchus mykiss) predict dioxin-like toxicity in early life stages? Mar. Environ. Res. 2000, 50 (1-5), 308-309.

(9) Guo, Y.; Senthilkumar, K.; Alomirah, H.; Moon, H. B.; Minh, T. B.; Mohd, M. A.; Nakata, H.; Kannan, K. Concentrations and profiles of urinary polycyclic aromatic hydrocarbon metabolites (OH-PAHs) in several Asian countries. Environ. Sci. Technol. 2013, 47 (6), 29322938.

(10) Richard, P.; Moody, I. C. Dermal exposure to environmental contaminants in the Great Lakes. Environ. Health Perspect. 1995, 103 (Supplement 9), 103-114.

(11) Blokker, E. J. M.; van de Ven, B. M.; de Jongh, C. M.; Slaats, P. G. G. (Nellie). Health Implications of PAH Release from Coated Cast Iron Drinking Water Distribution Systems in the Netherlands. Environ. Health Perspect. 2013, 121 (5), 600-606.

(12) Maskaoui, K.; Zhou, J.; Hong, H.; Zhang, Z. Contamination by polycyclic aromatic hydrocarbons in the Jiulong River Estuary and Western Xiamen Sea, China. Environ. Pollut. 2002, 118 (1), 109-122.

(13) Huang, L.; Wang, C.; Zhang, Y.; Wu, M.; Zuo, Z. Phenanthrene causes ocular developmental toxicity in zebrafish embryos and the possible mechanisms involved. J. Hazard. Mater. 2013, 261, 172-180.

(14) Huang, L.; Xi, Z.; Wang, C.; Zhang, Y.; Yang, Z.; Zhang, S.; Chen, Y.; Zuo, Z. Phenanthrene exposure induces cardiac hypertrophy via reducing miR-133a expression by DNA methylation. Sci. Rep. 2015, 2016 (6), 20105.

(15) Sun, L.; Zuo, Z.; Luo, H.; Chen, M.; Zhong, Y.; Chen, Y.; Wang, C. Chronic Exposure to Phenanthrene Influences the Spermatogenesis of Male Sebastiscus marmoratus: U-Shaped Effects and the Reason for Them. Environ. Sci. Technol. 2011, 45 (23), 10212-10218.

(16) Valdez Domingos, F. X.; Oliveira Ribeiro, C. A.; Pelletier, E.; Rouleau, C. Tissue Distribution and Depuration Kinetics of Waterborne 14 C-labeled Light PAHs in Mummichog (Fundulus heteroclitus). Environ. Sci. Technol. 2011, 45 (7), 2684-2690.

(17) Karami, A.; Romano, N.; Hamzah, H.; Simpson, S. L.; Yap, C. K. Acute phenanthrene toxicity to juvenile diploid and triploid African catfish (Clarias gariepinus): Molecular, biochemical, and histopathological alterations. Environ. Pollut. 2016, 212, 155-165.

(18) Yin, Y.; Jia, H.; Sun, Y.; Yu, H.; Wang, X.; Wu, J.; Xue, Y. Bioaccumulation and ROS generation in liver of Carassius auratus, exposed to phenanthrene. Comp. Biochem. Physiol., Part C: Toxicol. Pharmacol. 2007, 145 (2), 288-293.

(19) Bartel, D. P. MicroRNAs: Genomics, Biogenesis, Mechanism, and Function. Cell 2004, 116 (2), 281-297.

(20) Lin, S.; Gregory, R. I. MicroRNA biogenesis pathways in cancer. Nat. Rev. Cancer 2015, 15 (6), 321-333.

(21) Vrijens, K.; Bollati, V.; Nawrot, T. S. MicroRNAs as potential signatures of environmental exposure or effect: A systematic review. Environ. Health Perspect. 2015, 123 (5), 399-411.

(22) Calin, G. a.; Cimmino, A.; Fabbri, M.; Ferracin, M.; Wojcik, S. E.; Shimizu, M.; Taccioli, C.; Zanesi, N.; Garzon, R.; Aqeilan, R. I.; Alder, H.; Volinia, S.; Rassenti, L.; Liu, X.; Liu, C. -g.; Kipps, T. J.; Negrini, M.; Croce, C. M. MiR-15a and miR-16-1 cluster functions in human leukemia. Proc. Natl. Acad. Sci. U. S. A. 2008, 105 (13), 51665171.

(23) Zhu, L.; Gao, J.; Huang, K.; Luo, Y.; Zhang, B.; Xu, W. miR-34a screened by miRNA profiling negatively regulates $\mathrm{Wnt} / \beta$-catenin signaling pathway in Aflatoxin B1 induced hepatotoxicity. Sci. Rep. 2015, 5 (1), 16732.

(24) Wang, Y.; Tang, N.; Hui, T.; Wang, S.; Zeng, X.; Li, H.; Ma, J. Identification of endogenous reference genes for RT-qPCR analysis of plasma microRNAs levels in rats with acetaminophen-induced hepatotoxicity. J. Appl. Toxicol. 2013, 33 (11), 1330-1336.

(25) Olive, V.; Bennett, M. J.; Walker, J. C.; Ma, C.; Jiang, I.; Cordon-Cardo, C.; Li, Q. J.; Lowe, S. W.; Hannon, G. J.; He, L. miR19 is a key oncogenic component of mir-17-92. Genes Dev. 2009, 23, 2839-2849.

(26) Liang, X.; Zha, J. Toxicogenomic applications of Chinese rare minnow (Gobiocypris rarus) in aquatic toxicology. Comp. Biochem. Physiol., Part D: Genomics Proteomics 2016, 19, 174-180.
(27) Mu, J.; Wang, J.; Jin, F.; Wang, X.; Hong, H. Comparative embryotoxicity of phenanthrene and alkyl-phenanthrene to marine medaka (Oryzias melastigma). Mar. Pollut. Bull. 2014, 85, 505-515.

(28) Chen, R.; Liu, C.; Yuan, L.; Zha, J.; Wang, Z. 2, 4-Dichloro-6nitrophenol, a photonitration product of 2, 4-dichlorophenol, caused anti-androgenic potency in Chinese rare minnows (Gobiocypris rarus). Environ. Pollut. 2016, 216, 591-598.

(29) Chan, P. K.; Cheng, S. H. Cadmium-induced ectopic apoptosis in zebrafish embryos. Arch. Toxicol. 2003, 77 (2), 69-79.

(30) Zha, J.; Hong, X.; Rao, H.; Yuan, L.; Wang, Z.; Kumaran, S. S. Benzo(a)pyrene-induced a mitochondria-independent apoptosis of liver in juvenile Chinese rare minnows (Gobiocypris rarus). Environ. Pollut. 2017, 231, 191-199.

(31) Ghosh, C.; Zhou, Y. L.; Collodi, P. Derivation and characterization of a zebrafish liver cell line. Cell Biol. Toxicol. 1994, 10 (3), 167-176.

(32) Perry, S. W.; Norman, J. P.; Barbieri, J.; Brown, E. B.; Harris, A. Mitochondrial membrane potential probes and the proton gradient: a practical usage guide. BioTechniques 2011, 50 (2), 98-115.

(33) Yuan, L.; Li, J.; Zha, J.; Wang, Z. Targeting neurotrophic factors and their receptors, but not cholinesterase or neurotransmitter, in the neurotoxicity of TDCPP in Chinese rare minnow adults (Gobiocypris rarus). Environ. Pollut. 2016, 208, 670-677.

(34) He, J.; Chen, Q.; Wei, Y.; Jiang, F.; Yang, M.; Hao, S.; Guo, X.; Chen, D.; Kang, L. MicroRNA-276 promotes egg-hatching synchrony by up-regulating brm in locusts. Proc. Natl. Acad. Sci. U. S. A. 2016, 113 (3), 584-589.

(35) Hong, X.; Qin, J.; Chen, R.; Yuan, L.; Zha, J.; Wang, Z. Identification and characterization of novel and conserved microRNAs in several tissues of the Chinese rare minnow (Gobiocypris rarus) based on illumina deep sequencing technology. BMC Genomics 2016, 17 (1), 283.

(36) Schmittgen, T. D.; Livak, K. J. Analyzing real-time PCR data by the comparative CT method. Nat. Protoc. 2008, 3 (6), 1101-1108.

(37) Morgan, C. E.; Meagher, J. L.; Levengood, J. D.; Delproposto, J.; Rollins, C.; Stuckey, J. A.; Tolbert, B. S. The First Crystal Structure of the UP1 Domain of hnRNP A1 Bound to RNA Reveals a New Look for an Old RNA Binding Protein. J. Mol. Biol. 2015, 427 (20), 32413257.

(38) Li, N.; Jiang, W.; Ma, M.; Wang, D.; Wang, Z. Chlorination byproducts of bisphenol $\mathrm{A}$ enhanced retinoid $\mathrm{X}$ receptor disrupting effects. J. Hazard. Mater. 2016, 320, 289-295.

(39) Ha, M.; Kim, V. N. Regulation of microRNA biogenesis. Nat. Rev. Mol. Cell Biol. 2014, 15 (8), 509-524.

(40) Guil, S.; Cáceres, J. F. The multifunctional RNA-binding protein hnRNP A1 is required for processing of miR-18a. Nat. Struct. Mol. Biol. 2007, 14 (7), 591-596.

(41) Zhang, Y.; Tao, S. Global atmospheric emission inventory of polycyclic aromatic hydrocarbons (PAHs) for 2004. Atmos. Environ. 2009, 43 (4), 812-819.

(42) Kim, K.-H.; Jahan, S. A.; Kabir, E.; Brown, R. J. C. A review of airborne polycyclic aromatic hydrocarbons (PAHs) and their human health effects. Environ. Int. 2013, 60, 71-80.

(43) Barron, M. G.; Carls, M. G.; Heintz, R; Rice, S. D. Evaluation of fish early life-stage toxicity models of chronic embryonic exposures to complex polycyclic aromatic hydrocarbon mixtures. Toxicol. Sci. 2004, 78 (1), 60-67.

(44) Mu, J. li; Wang, X. hong; Jin, F.; Wang, J. ying; Hong, H. sheng The role of cytochrome P4501A activity inhibition in three- to fiveringed polycyclic aromatic hydrocarbons embryotoxicity of marine medaka (Oryzias melastigma). Mar. Pollut. Bull. 2012, 64 (7), 14451451.

(45) Song, M. K.; Kim, Y. J.; Song, M.; Ryu, J. C. Gene expression analysis identifies potential biomarkers of phenanthrene in human hepatocytes (HepG2). Toxicol. Environ. Health Sci. 2011, 3 (1), 3038.

(46) Wu, L. L.; L Chen, Y. L.; Zhang, J. L. Effects of phenanthrene toxicity on histopathology of Brachydanio rerio gill and liver. Chin. J. Ecol. 2007, 26 (5), 688-692. 
(47) Shi, Y. Mechanisms of Caspase Activation and Inhibition during Apoptosis. Mol. Cell 2002, 9 (3), 459-470.

(48) Ouyang, L.; Shi, Z.; Zhao, S.; Wang, F. T.; Zhou, T. T.; Liu, B.; Bao, J. K. Programmed cell death pathways in cancer: a review of apoptosis, autophagy and programmed necrosis. Cell Proliferation 2012, 45 (15), 487-498.

(49) van Delft, J. H. M.; Mathijs, K.; Staal, Y. C. M.; van Herwijnen, M. H. M.; Brauers, K. J. J.; Boorsma, A.; Kleinjans, J. C. S. Time series analysis of benzo[a]pyrene-induced transcriptome changes suggests that a network of transcription factors regulates the effects on functional gene sets. Toxicol. Sci. 2010, 117 (2), 381-392.

(50) Kim, S. J.; Ko, C. B.; Park, C.; Kim, B. R.; Sung, T. H.; Koh, D. H.; Kim, N. S.; Oh, K. J.; Chung, S. Y.; Park, R. p38 MAP kinase regulates benzo(a)pyrene-induced apoptosis through the regulation of p53 activation. Arch. Biochem. Biophys. 2005, 444 (2), 121-129.

(51) Drukteinis, J. S.; Medrano, T.; Ablordeppey, E. A.; Kitzman, J. M.; Shiverick, K. T. Benzo[a]pyrene, but Not 2,3,7,8-TCDD, Induces G2/M Cell Cycle Arrest, p21CIP1 and p53 Phosphorylation in Human Choriocarcinoma JEG-3 Cells: A Distinct Signaling Pathway. Placenta 2005, 26 (Suppl), S87-S95.

(52) Bendjennat, M.; Boulaire, J.; Jascur, T.; Brickner, H.; Barbier, V.; Sarasin, A.; Fotedar, A.; Fotedar, R. UV irradiation triggers ubiquitindependent degradation of p21 WAF1 to promote DNA repair. Cell 2003, 114 (5), 599-610.

(53) Hollander, M. C.; Kovalsky, O.; Salvador, J. M.; Kim, K. E.; Patterson, A. D.; Haines, D. C.; Fornace, A. J. Dimethylbenzanthracene carcinogenesis in Gadd45a-null mice is associated with decreased DNA repair and increased mutation frequency. Cancer Res. 2001, 61 (6), 2487-2491.

(54) Mogilyansky, E.; Rigoutsos, I. The miR-17/92 cluster: a comprehensive update on its genomics, genetics, functions and increasingly important and numerous roles in health and disease. Cell Death Differ. 2013, 20 (12), 1603-1614.

(55) Lima, R. T.; Busacca, S.; Almeida, G. M.; Gaudino, G.; Fennell, D. a.; Vasconcelos, M. H. MicroRNA regulation of core apoptosis pathways in cancer. Eur. J. Cancer 2011, 47 (2), 163-174.

(56) Munoz-garrido, P.; Marzioni, M.; Hijona, E.; Bujanda, L.; Banales, J. M. MicroRNAs in Liver Diseases. In MicroRNAs in Medicine; John Wiley \& Sons, Inc.: Hoboken, NJ, 2013; pp 509-522.

(57) Aguda, B. D.; Kim, Y.; Piper-Hunter, M. G.; Friedman, A.; Marsh, C. B. MicroRNA regulation of a cancer network: Consequences of the feedback loops involving miR-17-92, E2F, and Myc. Proc. Natl. Acad. Sci. U. S. A. 2008, 105 (50), 19678-19683.

(58) Olive, V.; Sabio, E.; Bennett, M. J.; De Jong, C. S.; Biton, A.; McGann, J. C.; Greaney, S. K.; Sodir, N. M.; Zhou, A. Y.; Balakrishnan, A.; et al. A component of the mir-17-92 polycistronic oncomir promotes oncogene-dependent apoptosis. eLife 2013, 2, e00822.

(59) Guillouzo, A. Liver cell models in in vitro toxicology. Environ. Health Perspect. 1998, 106 (Suppl.2), 511-532.

(60) Leung, C. W. T.; Hong, Y.; Chen, S.; Zhao, E.; Lam, J. W. Y.; Tang, B. Z. A Photostable AIE Luminogen for Specific Mitochondrial Imaging and Tracking. J. Am. Chem. Soc. 2013, 135 (1), 62-65.

(61) Ly, J. D.; Grubb, D. R.; Lawen, A. The mitochondrial membrane potential $(\Delta \psi \mathrm{m})$ in apoptosis; an update. Apoptosis 2003, $8(2), 115-$ 128.

(62) Ameres, S. L.; Zamore, P. D. Diversifying microRNA sequence and function. Nat. Rev. Mol. Cell Biol. 2013, 14 (8), 475-488.

(63) Fujiya, M.; Konishi, H.; Kamel, M. K. M.; Ueno, N.; Inaba, Y.; Moriichi, K.; Tanabe, H.; Ikuta, K.; Ohtake, T.; Kohgo, Y. microRNA$18 \mathrm{a}$ induces apoptosis in colon cancer cells via the autophagolysosomal degradation of oncogenic heterogeneous nuclear ribonucleoprotein A1. Oncogene 2014, 33 (40), 4847-4856.

(64) Michlewski, G.; Guil, S.; Semple, C. A.; Cáceres, J. F. Posttranscriptional Regulation of miRNAs Harboring Conserved Terminal Loops. Mol. Mol. Cell 2008, 32 (3), 383-393.

(65) Michlewski, G.; Guil, S.; Cáceres, J. F. Stimulation of pri-miR18a processing by hnRNP A1. Adv. Exp. Med. Biol. 2010, 700, 28-35. 